\title{
Attribute-aware Semantic Segmentation of Road Scenes for Understanding Pedestrian Orientations
}

\author{
M. D. Sulistiyo ${ }^{1,2}$, Y. Kawanishi ${ }^{1}$, D. Deguchi ${ }^{3}$, T. Hirayama $^{4}$, I. Ide ${ }^{1}$, J. Y. Zheng ${ }^{1,5}$, H. Murase ${ }^{1}$
}

\begin{abstract}
Semantic segmentation is an interesting task to many deep learning researchers for scene understanding. However, recognizing details about object's attributes can be more informative and also helpful for a better scene understanding in intelligent vehicle uses. This paper introduces a method for simultaneous semantic segmentation and pedestrian attributes recognition. A modified dataset built on top of the Cityscapes dataset is created by adding attribute classes corresponding to pedestrian orientation attributes. The proposed method extends the SegNet model and is trained by using both the original and the attribute-enriched datasets. Based on an experiment, the proposed attribute-aware semantic segmentation approach shows the ability to slightly improve the performance on the Cityscapes dataset, which is capable of expanding its classes in this case through additional data training.
\end{abstract}

\section{INTRODUCTION}

In recent years, computer vision by deep learning have become popular for various complex tasks. One of the most interesting topics is the semantic segmentation, which is a more complex task compared to simple image segmentation. Semantic segmentation can be addressed as pixel-wise labeling and simultaneous classification of pixels in the input image into predefined classes. Semantic segmentation with deeplearning approaches have been widely utilized for the purposes such as indoor and outdoor scene understanding as well as applications like autonomous vehicle sensing [1] and robotics navigation [2]. For instance, from a road scene, a model trained for semantic segmentation can inform an autonomous vehicle of the objects around before the vehicle determines its action.

Many studies on this task have been conducted so far. A Fully Convolutional Network was introduced for the semantic segmentation task as an end-to-end trained model [3]. Almost in the same period, SegNet with a different network architecture was also published [1]. It was then modified into Bayesian SegNet and obtained better performance by adding a probabilistic element [4]. Later on, the Pyramid Scene Parsing network (PSPNet) [5] outperformed other existing methods and achieved a state-of-the-art performance in mean IoU score for PASCAL VOC 2012 [6] and Cityscapes datasets [7]. Very recently, Mask R-CNN presented a conceptual framework for

\footnotetext{
${ }^{1}$ Mahmud Dwi Sulistiyo, Yasutomo Kawanishi, Ichiro Ide, Hiroshi Murase are with Graduate School of Informatics, Nagoya University, Japan \{kawanishi, ide, murase\} @i.nagoya-u.ac.jp

${ }^{2}$ Mahmud Dwi Sulistiyo is also with School of Computing, Telkom University, Indonesia mahmuddwis atelkomuniversity.ac.id

${ }^{3}$ Daisuke Deguchi is with Information Strategy Office, Nagoya University, Japan ddeguchi@nagoya-u.jp

${ }^{4}$ Takatsugu Hirayama is with Institutes of Innovation for Future Society, Nagoya University, Japan takat sugu.hirayama@nagoya-u.jp

${ }^{5}$ Jiang Yu Zheng is with Dept. of Computer Science, Indiana UniversityPurdue University Indianapolis, USA jzheng@iupui.edu
}

instance-level segmentation, which combined object detection and semantic segmentation tasks. The approach outperformed some previous methods for several challenges [8]. Reference [9] provides a summary of popular deep neural networks that were adopted to solve the semantic segmentation.

However, the existing semantic segmentation methods only recognize an object's name as its class, e.g., road, building, car, person, and so on, while for autonomous vehicles, additional information describing a particular object in detail such as its attributes could help the better understanding of scenes. Therefore, this paper proposes an attribute-aware semantic segmentation method, which is a more difficult challenge. Yet we can obtain better evaluation results if the process is successfully performed. With simultaneous recognition and segmentation tasks, it can enhance environment perception tasks in Intelligent Transportation Systems that need precision such as parking, road following, pedestrian detection, action identification, and localization.

Here we chose pedestrian as the target class, out of other classes like vehicle and traffic signs, as it has many attributes to be explored. Since a pedestrian is one of the most important moving objects in a street scene, it should be useful for assisting an autonomous vehicle. In this paper, we particularly consider the body orientation of a pedestrian as the attribute and divide it into a predefined number of classes. In the autonomous vehicle system, being informed of pedestrian orientations can be helpful in collision avoidance. Although only the pedestrian body orientation was added as a new semantic information, it can give hint and insight on other object attributes when the method is extended.

The contributions of this paper are as follows:

1) We introduce a new concept of attribute-aware semantic segmentation that is general and possible to be applied to various tasks.

2) We design an attributes-dependent loss function that is modified from original one for increasing the number of classes regarding the attribute-aware concept.

3) We modify the annotation of Cityscapes dataset for the attribute-aware semantic segmentation task.

4) By extending the Cityscapes to include additional attribute classes, we achieved a better performance of semantic segmentation.

The rest of paper will discuss more about related work in Sec. II, our proposed idea and its implementation in Sec. III, and our modified dataset annotation in Sec. IV. Experimental results and discussions are presented in Sec. V followed with conclusion. 


\section{RELATED WORKS}

Many methods in deep learning are trying to solve the semantic segmentation task [1], [3]-[5], [8]. Among them, SegNet was motivated mainly by road-scene understanding. It is a deep neural network architecture consisting of convolutional encoder-decoder layers as shown in Figure 1.

Meanwhile, studies related to the pedestrian attribute recognition task have also been widely conducted. References [10] and [11] introduced a new dataset and presented a benchmark performance by an SVM-based method for pedestrian attribute recognition in far-view surveillance scenes and proposed an alternative approach for an improved attribute inference. To improve real-time applications, the Richly Annotated Pedestrian (RAP) dataset was proposed in reference [12]. It reported that attributes such as viewpoints, occlusions, and body parts information could help recognize pedestrian attributes in real applications.

Some related datasets with annotated images are publicly available for the challenges of semantic segmentation with various environments and lighting conditions. A number of datasets are used in reference [9] for a review on deep-learning techniques. Cityscapes is a benchmark suit and a large-scale dataset to train and test methods for pixel-level and instancelevel semantic labeling. The dataset was highly motivated by the need of semantic urban-scene understanding applications. Images were collected to cover various street conditions in a number of different cities. It was initially published as an ongoing project in reference [13] and finally completed in reference [7]. Many studies are addressed and challenged using this dataset for comparison to other existing datasets. It is the largest and the most diverse dataset of street scenes with highquality coarse annotations [7].

For standalone objects such as bicycles, riders, and traffic signs, the network can be trained to pick up their unique texture and local shape as the features for their identification. These features through filtering at different layers are still very stable to the final layer. For extending regions such as road, tree, cars, and sidewalk, the network propagates more position and shape information through linear combination of coefficients and max-pooling mechanism in the network according to tagged samples in the learning process. This "propagation" of segment $i d$ stops at strong edges but may fail to line out a uniformed region if there is no clear edge cue; the segmented region is thus noisy.

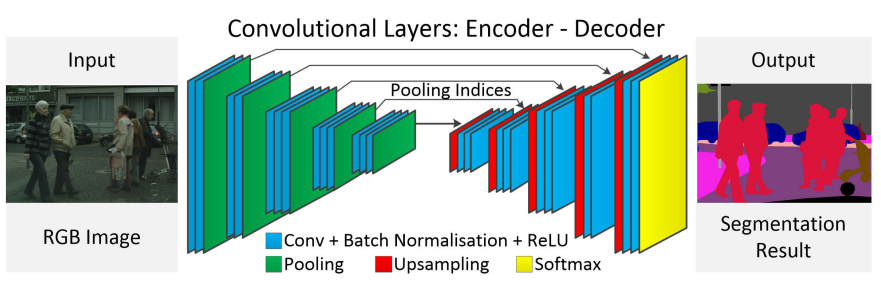

Fig. 1. SegNet's network architecture [1].

\section{Attribute-AwAre SegNet}

\section{A. Introducing Attributes-grouping Loss Function}

We propose an attribute-aware semantic segmentation concept to improve the semantic segmentation task by simultaneously obtaining per-pixel class label as well as the attribute information of a particular object. Basically, it extends the classification from an object class to its sub-classes. For example, in addition to classifying vegetation, car, and pedestrian, the classifier should also distinguish between types of vegetation, types of car, and even between male and female pedestrians. We assume that some particular classes have disjoint subclasses divided according to some attributes. We introduce this primary concept by modifying the neural network's structure and loss function so as to enrich the object understanding by their attributes.

In the current work, we use SegNet as the baseline model since it is a low-cost design in terms of memory usage and computational time during inference [1], while maintaining a better performance than several comparable methods. Regarding the loss function, there are several forms for existing deep neural network models; and particularly, SegNet uses a crossentropy loss function in its Softmax layer to produce the output [1], which is defined as follows:

$$
L\left(y, y^{\prime}\right)=-\sum_{i} y_{i} \log y_{i}^{\prime},
$$

where $y$ is the actual output of the network, and $y^{\prime}$ is the target output given in the dataset. $N$ is the number of training samples, so $y_{i}$ and $y_{i}^{\prime}$ are the actual and the target outputs of the $i$-th sample.

Considering that the attributed classes are actually derived from an object class, we extend the class loss function by adding a loss calculation unit for its attribute. The modified loss function can be written as follows:

$$
\begin{gathered}
L^{\prime}\left(y, y^{\prime}\right)=L_{c}+L_{a}, \\
L_{c}=-\beta_{c} \sum_{i} y_{i_{c}} \log y_{i_{c}}^{\prime}, \\
L_{a}=-\beta_{a} \sum_{i} y_{i_{a}} \log y_{i_{a}}^{\prime} . \\
\beta_{c}+\beta_{a}=1 .
\end{gathered}
$$

The class loss and attribute loss functions are denoted as $L_{c}$ and $L_{a}$, respectively. We furthermore introduce parameters $\beta_{c}$ and $\beta_{a}$ as the weights for both class and attribute losses, respectively. They are constant numbers ranging from 0 to 1 to determine the influence level of each loss score for the combined loss function score. For classes with no attribute, $\beta_{c}$ and $\beta_{a}$ are set to be 1 and 0 , respectively. However, determining those values between 0 and 1 is another issue to optimized in the future.

\section{B. Implementation of the Proposed Method}

Weighting the class loss as well as the attribute loss with appropriate values will efficiently solve this task. In addition, setting different values for $\beta_{c}$ and $\beta_{a}$ will also affect the 
network implementation, i.e. the network output units might be constructed in some different levels. On the other hand, assigning the parameters $\beta_{c}$ with 0 and $\beta_{a}$ with 1 implies that in the network implementation, there will be additional subclasses in the same level of the output layer. This means the attribute-aware semantic segmentation task will be represented in a so-called 'flat classifier' instead of hierarchical one [19].

For example, let's consider a set of classes $C=\{X, Y, Z\}$ and let class $X$ have a set of subclasses $A_{X}=\{x, y, z\}$ divided by attribute $A$; while classes $Y$ and $Z$ do not have any attribute. Note that $|C|$ is the number of classes, while $\left|A_{X}\right|$ is the number of sub-classes determined by attributes. Thus, the number of output units in the final connected layer will be $\left(|C|+\left|A_{X}\right|-1\right)$. In this sample case, there will be five output units. By the proposed method, the network will be trained using target classes of $C^{\prime}=C \cup A_{X}$. In the inference phase, the output of the trained model can be either $\left|C^{\prime}\right|$ number of classes, consisting of object and attribute classes, in a flat model, or just $|C|$ by integrating the attribute classes into one object class in a hierarchical model. In the sampling case, the first model output will be $\{x, y, z, Y, Z\}$ and the second alternative output will be $\{X, Y, Z\}$ after combining $\{x, y, z\}$ into class $X$. Figure 2 depicts this proposed concept for the sample case compared to the basic concept.

In addition to the structure of network, typically for the semantic segmentation, it is necessary to weight the losses differently between classes, since the sample numbers of classes (road, sidewalk, car, pedestrian, etc.) usually have a large variance [1] in a training set. Therefore, class balancing by calculating the loss weight for each class is needed, including the attribute classes since they are treated as object classes as well. The idea is to simply put more attention or weight on to the classes with pixels appearing infrequently, and give a smaller weight to a class that has a high pixel frequency. We use a median frequency balancing strategy by computing the pixel frequency of each class in the training set. The balancing factor on each class, denoted by $\alpha_{c}$, is obtained from the median of all class frequencies divided by its own class frequency [14]. Here, $\alpha_{c}$ is defined as follows:

$$
\alpha_{c}=\frac{\text { median_freq }}{\text { freq }_{c}}
$$

where freq ${ }_{c}$ is the number of pixels in class $c$ divided by the
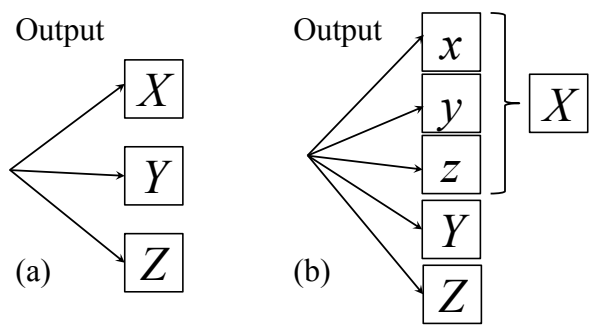

Fig. 2. Network structure comparison of approaches. (a) Basic output units for object level semantic segmentation; (b) Proposed concept for attribute-aware semantic segmentation. total number of pixels in images where class $c$ is present, while median_freq is the median value of all freq ${ }_{c}$. This formula implies that the more frequent a pixel class occurs in the training set, the less loss weight it gains (the value is less than 1).

\section{ATtRibute-EnRICHED Cityscapes Dataset}

\section{A. Cityscapes Dataset and CityPersons}

The Cityscapes dataset contains a total of 5,000 images, recorded in streets from 50 different cities. It is divided into 2,975 images for training, 500 for validation, and 1,525 for testing. It also provides fine annotations for training and validation sets, while the test annotation is not publicly available for benchmarking purpose. There are also 20,000 extra images with coarse annotations but not used in our current work. The Cityscapes dataset is annotated with 34 different class labels encoded by label_ID. However, since some labels are objects such as border, ground, and so on, which are unnecessary here and can be ignored, we use a simpler encoding version, namely train_ID, that reduces the task complexity into 19 class labels. The Cityscapes recommends users to use train_ID instead of label_ID during the training phase.

Our study also makes use of the CityPersons annotation [15], which is a new set of person annotations with high quality bounding boxes. It was built upon the Cityscapes dataset and provides better data for improved pedestrian detection. The CityPersons bounding box annotation is utilized in our work to easily extract each pedestrian instance before conducting pedestrian orientation annotation on the dataset.

The CityPersons dataset explains various conditions of a pedestrian class based on the person types and occlusion levels. Person types in this dataset consist of pedestrian, rider, sitting person, and unusual posture; while the occlusion levels range from 0.0 to 0.9 . The occlusion level 0.0 indicates the person has no occlusion, while 0.9 indicates he/she is almost completely occluded by some objects. The bounding box annotation is also well-aligned with the dimension ratio of 0.41 . The dataset provides annotations for 19,654 persons in the training set and 3,938 in the validation set [15]. In our work, we extract only pedestrian-type person images with occlusion level below 0.1 .

\section{B. Pedestrian Orientation Annotation}

To add new annotations to each extracted Cityscapes pedestrian, we used the Pedestrian Direction Classification (PDC) dataset from reference [6] as an image reference. We also referred to reference [16] for the orientation rules. The annotation process to modify Cityscapes dataset is as follows.

1) Extract single pedestrian images that are bounding boxes from Cityscapes images; CityPersons annotation is used to locate such single person pixels.

2) Filter the extracted persons considering the type and the occlusion level; Here, the person type should be pedestrian with a maximum occlusion level of 0.1 . 


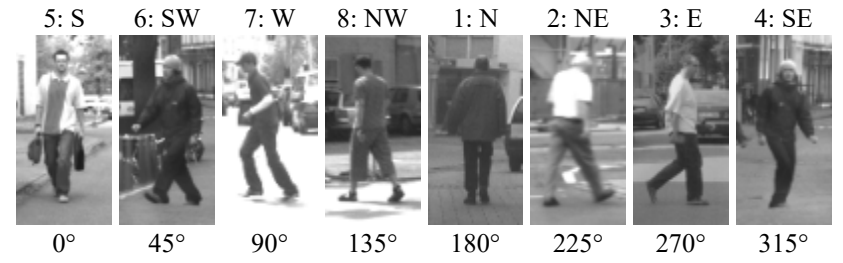

Fig. 3. References for annotating pedestrian directions [16].
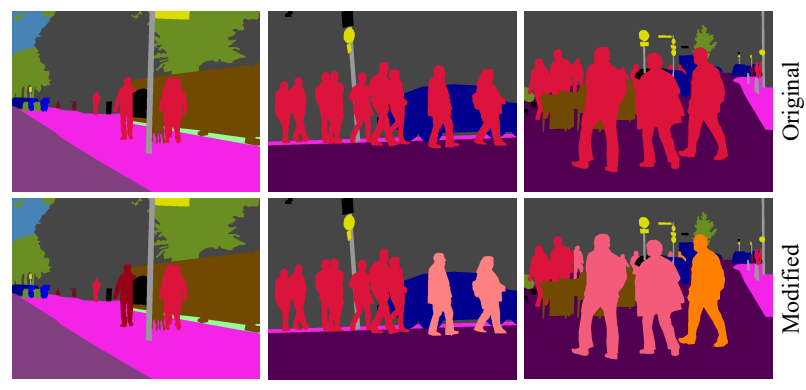

Fig. 4. New colored ground-truth examples with pedestrian orientations compared to original ones.

3) The pedestrian image collection is then manually annotated into orientation classes; The CityPersons annotation is then modified by adding the attribute class labels to the bounding boxes. We refer to references [6] and [16] for this process.

4) Change the person label in Cityscapes into one of eight person classes based on the orientation; This process adds a new Cityscapes pixel annotation.

5) Because of the inaccurate location of bounding boxes on pedestrians, we manually refine the drop-off regions from the boxes (e.g., feet), and add-in regions to the boxes from other pedestrians further.

The pedestrian orientation annotation process yielded 2,216 and 458 well-aligned pedestrian images for training and validation images, respectively. Table I shows the number of pedestrians annotated in each orientation class. We annotated cropped pedestrian images one-by-one solidly. Our orientation annotation is slightly different to that in reference [16], where we used label ' 1 ' to denote direction $\mathrm{N}$, '2' for $\mathrm{NE}$, and so on until ' 8 ' for NW, respectively, as shown in Figure 3.

The class number was increased from 19 to 27 since we replaced class 'person' with 9 attribute classes ( 8 orientation classes and 1 class for unknown orientation). Figure 4 depicts samples of new ground-truth images compared to the original ones after additional annotations were applied. In the original ground-truth, all pedestrians had the same color, while in the modified ones, one pedestrian might have a different color with another.

\section{EXPERIMENTS AND DISCUSSION}

\section{A. Experiment Design and Implementation}

The experiments are conducted to 1) train the proposed model for attribute-aware semantic segmentation, 2) analyze
TABLE I

Class Distributions of Annotated Pedestrian Orientations

\begin{tabular}{|l|r|r|r|r|r|r|r|r|}
\hline \multicolumn{1}{|c|}{ Set } & \multicolumn{1}{c|}{$\mathbf{1}$} & \multicolumn{1}{c|}{$\mathbf{2}$} & \multicolumn{1}{c|}{$\mathbf{3}$} & $\mathbf{4}$ & \multicolumn{1}{c|}{$\mathbf{5}$} & \multicolumn{1}{c|}{} & \multicolumn{1}{c|}{$\mathbf{~}$} & \multicolumn{1}{c|}{8} \\
\hline Training & 466 & 131 & 327 & 126 & 545 & 115 & 334 & 172 \\
\hline Validation & 98 & 28 & 66 & 49 & 79 & 30 & 81 & 27 \\
\hline Total & $\mathbf{5 6 4}$ & $\mathbf{1 5 9}$ & $\mathbf{3 9 3}$ & $\mathbf{1 7 5}$ & $\mathbf{6 2 4}$ & $\mathbf{1 4 5}$ & $\mathbf{4 1 5}$ & $\mathbf{1 9 9}$ \\
\hline
\end{tabular}

whether the attribute-aware concept can help increase the semantic segmentation performance, and 3) compare the proposed method with the original SegNet on the same dataset.

The original SegNet is built on Caffe [17] implementation ${ }^{1}$. However, some adjustments are needed because it was built for another dataset with less complexity. After some technical modification on the code to satisfy the requirements of the Cityscapes dataset, we trained models using the training set and its annotations.

In this experiment, we compare two models: the original SegNet trained using the 19_label_set and the proposed attribute-aware SegNet trained using 27_label_set. The 19_label_set is a set of annotations based on the train_ID encoding scheme consisting of 19 labels to classify; while the 27_label_set is a set of annotation based on the train_ID encoding scheme with additional eight new labels for attribute annotated-person classes.

Training: We trained VGG 16 layers as the initial model to build our models with 50,000 steps for each training phase. Since we trained SegNet with a large dataset and image dimension, it was necessary to decrease the computational load to deal with the space complexity issue. The size of all images was reduced into half of the original image during the training phase, while the batch number was set to be 2 for smaller memory usage.

Testing: To evaluate the trained models, we used train_set and val_set images including their available annotations to calculate the performance metrics and compare the results. We also tested the original and the modified models using test_set and then submitted the results to the Cityscapes web site $^{2}$ for benchmarking purpose. Before submitting the results, all labels in output images should be converted into the original labels and all output images were resized into the original dimensions. Note that all pedestrian classes (a pedestrian with additional eight orientation labels) were combined into one pedestrian class label to comply with the Cityscapes labels and to compare the performances between the original SegNet and the proposed method.

\section{B. Performance Metric on Segmented CityScapes}

For internal comparison, we measured the performance of the models using two common metrics, which are global accuracy and mean Intersection over Union (IoU) [3], defined as follows:

$$
\text { glob_acc }=\frac{\text { true_predicted_pixels }}{\text { all_predicted_pixels }},
$$

\footnotetext{
${ }^{1}$ Publicly available at https://github.com/alexgkendall/caffe-segnet/. ${ }^{2}$ https://www.cityscapes-dataset.com/submit/.
} 


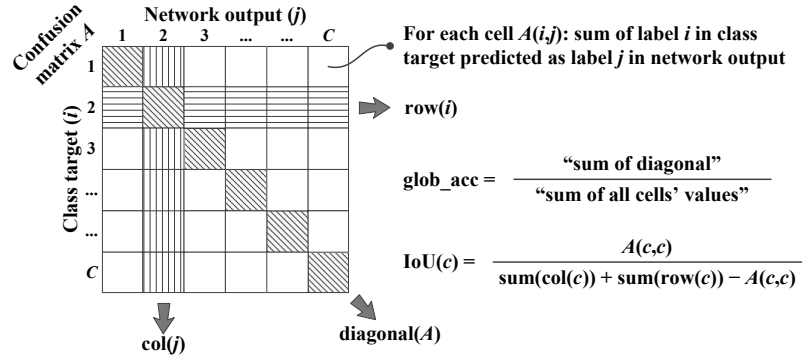

Fig. 5. Calculating global accuracy and IoU using confusion matrix.

$$
\begin{gathered}
\mathrm{IoU}_{c}=\frac{\mathrm{TP}_{c}}{\mathrm{TP}_{c}+\mathrm{FP}_{c}+\mathrm{FN}_{c}}, \\
\text { mean_IoU }=\frac{1}{C} \sum_{c} \mathrm{IoU}_{c} .
\end{gathered}
$$

where $\mathrm{IoU}_{c}$ is the IoU for class $c$ and $C$ is the number of classes, while TP, FP, and FN respectively denote the number of 'true positive', 'false positive', and 'false negative' pixels.

Based on Formulae 7, 8, and 9, we use a confusion matrix in the implementation to calculate the global accuracy and the mean IoU. Figure 5 illustrates the mechanism of the calculation.

\section{Results of Semantic Segmentation}

Table II shows the evaluation results for both original SegNet and proposed method. The original SegNet was trained using the Cityscapes dataset, while the proposed method was trained using our modified Cityscapes. We use the global accuracy (glob_acc) and the mean of IoU (mean_IoU) to measure the performance of both methods for the validation set. We also add the accuracy of pedestrian orientation (mean_IoU $\mathrm{U}_{\text {or }}$ ) computed exclusively for the proposed method. Meanwhile, Table III shows the per-class accuracy of our method on the testing set, which is benchmarked for comparison between existing methods for the Cityscapes dataset. The benchmarking system uses the IoU metric for measuring the performance. Besides, we can see the qualitative results of some output samples in Figure 6 selected from the validation set.

It is observable that the segmentation accuracy measured by $\mathrm{IoU}$ is still very low despite of the precise annotation of objects. This can be observed in the output results where boundaries of human, traffic sign, and so on are more close to blobs. It implies that the loss function has not picked up detailed differences on the boundary to teach the network in the supervised learning. The network is more semantic recognition than precise segmentation.

The time for segmentation after training is 1.7 frame per second, while the off-line network training certainly takes much more time lasting one day or so. The detection time is short to be considered in use on a slow moving vehicle.

TABLE II

Performance Evaluation on Validation Set

\begin{tabular}{|r|r||r|r|r|}
\hline \multicolumn{2}{|c||}{ Original SegNet } & \multicolumn{3}{c|}{ Proposed Method } \\
\hline glob_acc & mean_IoU & glob_acc & mean_IoU & mean_IoU or \\
\hline 84.4 & 42.3 & $\mathbf{8 6 . 3}$ & $\mathbf{5 0 . 3}$ & 27.8 \\
\hline
\end{tabular}

We use Titan X GPU in our current study for training and segmentation processes.

\section{Discussion on Accuracy and Data}

The experimental results show that the proposed method outperforms the original SegNet slightly. For both global accuracy and mean IoU, the detailed sub-classes identification has gained higher values than the original SegNet on average. This is achieved by the proposed concept and implementation of the attribute-aware semantic segmentation.

Applying a conventional semantic segmentation to a number of object classes usually implies in high deviation between classes. It causes the difficulty in classifying each pixel into a given set of labels, especially for a class with a low ratio in the training set. Meanwhile, the proposed method divides a particular class into some sub-classes that correspond to its attribute classes. This proposed concept implies that between-classes deviations are decreased. Accordingly, the semantic segmentation becomes easier. Thus, the concept to provide object attributes with additional semantic information improves the semantic segmentation. The training process can also start from an existing model to refine the detailed aspects or attributes in a shorter time.

On the other hand, the number of annotated pedestrians in this study is still limited. This can still be improved by collecting more attributed-object samples from the Cityscapes dataset. For example, adjusting the constraints of person type or occlusion level is necessary to increase the number of wellaligned pedestrian images for training and validation.

After the pedestrians are detected with orientation attributes, it is important to pay more intention on the persons facing road, because they are perhaps walking towards the vehicle path and the vehicles may prepare a sudden breaking. In the future, we will further target the motion information [18] in the driving video to feed in the recognition of pedestrian walking direction such that the segmentation of pedestrians can be more certain and robust.

\section{CONCLUSiON}

We proposed a new approach as the attribute-aware semantic segmentation to enrich recognition classes. A general concept was introduced to modify a deep neural network model to achieve this goal. We conducted an experiment using the Cityscapes dataset and the modified SegNet resulting in better performance as compared to the original one. We inserted the pedestrian body orientation as additional semantic information in the annotations to modify the Cityscapes dataset. This has proved that the proposed method not only performed an attribute recognition task but also enhanced the semantic segmentation. The proposed concept should be able to handle more object classes and attributes than pedestrians.

\section{ACKNOWLEDGMENT}

The first author would like to thank Indonesia's BUDI-LN scholarship for the generous supports to his doctoral study. Parts of this research were supported by MEXT, Grant-in-Aid for Scientific Research in Japan. 
TABLE III

Evaluation Results for the Testing SET Submitted to Cityscapes Benchmarking

\begin{tabular}{|c|c|c|c|c|c|c|c|c|c|c|c|c|c|c|c|c|c|c|c|c|}
\hline Method & 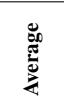 & ్ָ & 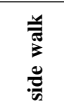 & & $\overline{\bar{\pi}}$ & 巳 & $\stackrel{\varrho}{\varrho}$ & 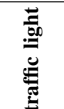 & 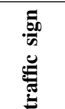 & 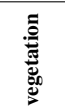 & 苞 & $\frac{2}{n}$ & 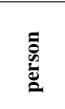 & $\stackrel{\Xi}{\overparen{\Xi}}$ & $\bar{z}$ & 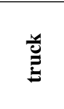 & 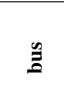 & 芯 & 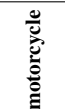 & 窇 \\
\hline Original SegNet & 44.25 & 85.78 & 51.78 & 75.48 & 23.36 & 26.46 & 26.22 & 33.48 & 35.30 & 83.85 & 48.66 & 86.93 & 57.37 & 29.35 & 76.61 & 5.30 & 27.15 & 0.03 & 19.68 & 47.99 \\
\hline Proposed Method & 45.92 & 89.94 & 54.64 & 73.76 & 20.50 & 17.95 & 29.70 & 33.53 & 34.97 & 84.34 & 51.14 & 86.51 & 59.58 & 37.96 & 81.90 & 11.98 & 26.76 & 1.75 & 31.59 & 43.91 \\
\hline
\end{tabular}
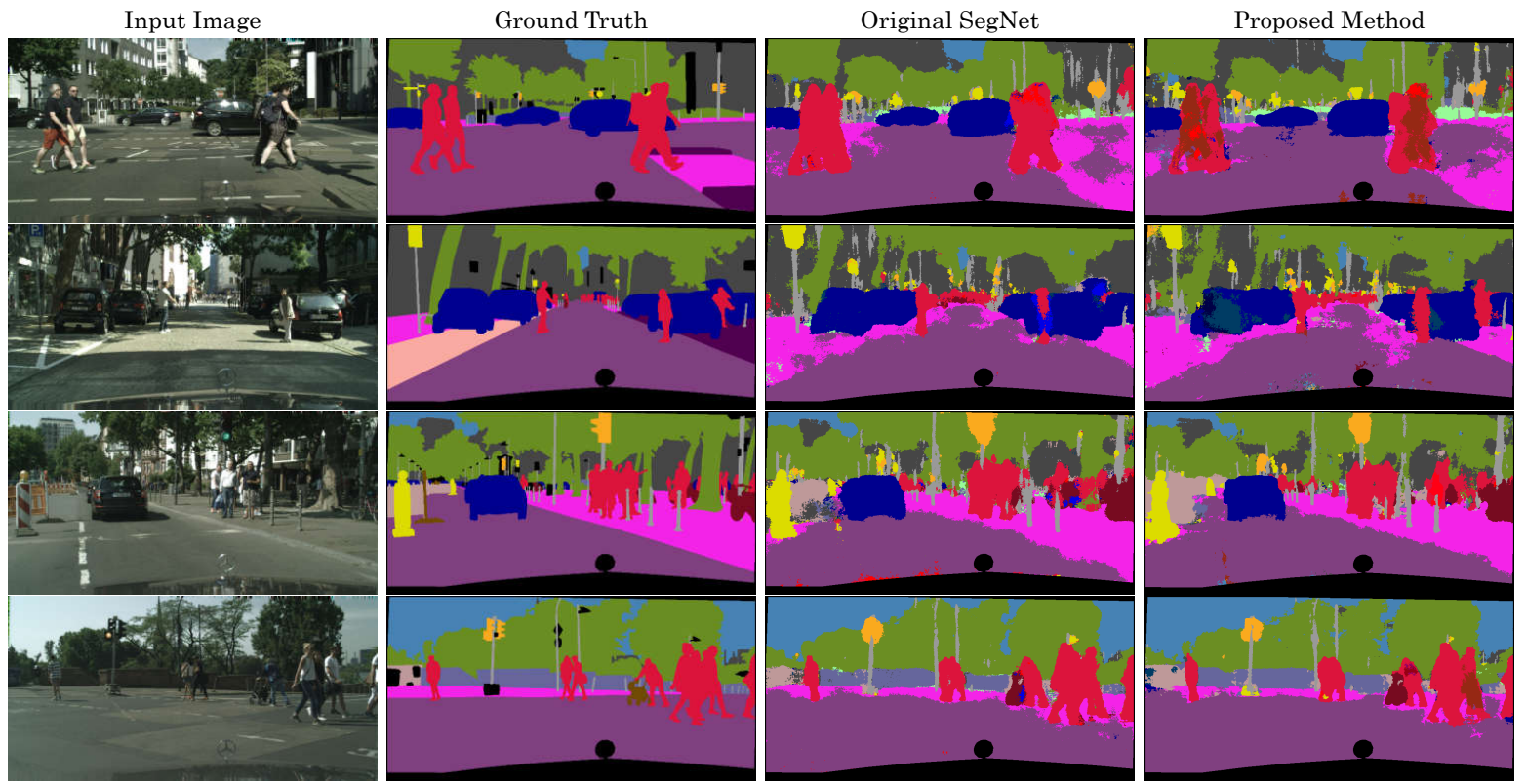

Fig. 6. Qualitative results for four input images selected from the validation set; The leftmost column is the input image, the second column is the fine ground-truth image from the Cityscapes dataset, the third column is the result from the SegNet model trained using the original dataset (19 classes), and the rightmost one is the result from the proposed method which is trained using a modified dataset (27 classes) and contains information of pedestrian orientation.

\section{REFERENCES}

[1] V. Badrinarayanan, A. Kendall, and R. Cipolla, "SegNet: A deep convolutional encoder-decoder architecture for image segmentation," in IEEE Trans. on Pattern Analysis and Machine Intelligence, vol. 39, no. 12, pp. 2481-2495, 1 Dec. 2017.

[2] B. J. Meyer and T. Drummond, "Improved semantic segmentation for robotic applications with hierarchical conditional random fields," in Proc. 2017 IEEE Int. Conf. on Robotics and Automation, 2017, pp. 5258-5265.

[3] J. Long, E. Shelhamer, and T. Darrell, "Fully convolutional networks for semantic segmentation," in Proc. 2015 IEEE Conf. on Computer Vision and Pattern Recognition, 2015, pp. 3431-3440.

[4] A. Kendall, V. Badrinarayanan, and R. Cipolla, "Bayesian SegNet: Model uncertainty in deep convolutional encoder-decoder architectures for scene understanding," arXiv preprint arXiv:1511.02680, 2015.

[5] H. Zhao, J. Shi, X. Qi, X. Wang, and J. Jia, "Pyramid scene parsing network," in Proc. IEEE Conf. on Computer Vision and Pattern Recognition, 2017, pp. 2881-2890.

[6] J. Tao and R. Klette, "Part-based RDF for direction classification of pedestrians, and a benchmark," in Computer Vision ACCV 2014 Workshops, ser. Lecture Notes in Computer Science, vol. 9009. Springer, 2014, pp. 418-432.

[7] M. Cordts, M. Omran, S. Ramos, T. Rehfeld, M. Enzweiler, R. Benenson, U. Franke, S. Roth, and B. Schiele, "The cityscapes dataset for semantic urban scene understanding," in Proc. 2016 IEEE Conf. on Computer Vision and Pattern Recognition, 2016, pp. 3213-3223.

[8] K. He, G. Gkioxari, P. Dollr, and R. Girshick, "Mask R-CNN," in Proc. IEEE Int. Conf. on Computer Vision, 2017, pp. 2980-2988.

[9] A. Garcia-Garcia, S. Orts-Escolano, S. Oprea, V. Villena-Martinez, and J. Garcia-Rodriguez, "A review on deep learning techniques applied to semantic segmentation," arXiv preprint arXiv:1704.06857, 2017.
[10] Y. Deng, P. Luo, C. C. Loy, and X. Tang, "Pedestrian attribute recognition at far distance" in Proc. 22nd ACM Int. Conf. on Multimedia, 2014, pp. 789-792.

[11] Y. Deng, P. Luo, C. C. Loy, and X. Tang, "Learning to recognize pedestrian attribute," arXiv preprint arXiv:1501.00901, 2015.

[12] D. Li, Z. Zhang, X. Chen, H. Ling, and K. Huang, "A richly annotated dataset for pedestrian attribute recognition," arXiv preprint arXiv:1603.07054, 2016.

[13] M. Cordts, M. Omran, S. Ramos, T. Scharwchter, M. Enzweiler, R. Benenson, U. Franke, S. Roth, and B. Schiele, "The cityscapes dataset," in Proc. 2015 IEEE Conf. on Computer Vision and Pattern Recognition Workshops, vol. 1, no. 2, 2015, p. 3 .

[14] D. Eigen and R. Fergus, "Predicting depth, surface normals and semantic labels with a common multi-scale convolutional architecture" in Proc. 2015 IEEE Int. Conf. on Computer Vision, 2015, pp. 2650-2658.

[15] S. Zhang, R. Benenson, and B. Schiele, "Citypersons: A diverse dataset for pedestrian detection," in Proc. 2017 IEEE Conf. on Computer Vision and Pattern Recognition, vol. 1, no. 2, 2017, p. 3.

[16] Y. Kawanishi, D. Deguchi, I. Ide, H. Murase, and H. Fujiyoshi, "Misclassification tolerable learning for robust pedestrian orientation classification," in Proc. 23rd Int. Conf. on Pattern Recognition, 2016, pp. 486-491.

[17] Y. Jia, E. Shelhamer, J. Donahue, S. Karayev, J. Long, R. Girshick, S. Guadarrama, and T. Darrell, "Caffe: Convolutional architecture for fast feature embedding," in Proc. 22nd ACM Int. Conf. on Multimedia, 2014, pp. 675-678.

[18] M. Kilicarslan, J. Y. Zheng, K. Raptis, "Pedestrain detection from motion," in Proc. 23rd Int. Conf. on Pattern Recognition, 2016, pp. $1857-1863$

[19] P. Meletis and G. Dubbelman, "Training of Convolutional Networks on Multiple Heterogeneous Datasets for Street Scene Semantic Segmentation" in Proc. 29th IEEE Intelligent Vehicle Symposium, 2018, pp. 1045-1050. 